\title{
COVID-19 na gestação: uma revisão integrativa
}

\author{
COVID-19 in pregnancy: an integrative review
}

COVID-19 en el embarazo: una revisión integradora

Monique Eva Vargas Cardoso ${ }^{1 \star}$, Gisele Cassão, Cristine Kasmirscki', Lúcia Fabiane da Silva Luz¹.

\section{RESUMO}

Objetivo: Identificar, sintetizar e analisar as literaturas referentes ao cuidado com COVID-19 na gestação. Métodos: Revisão integrativa nas bases de dados Medline e Epistemonikos utilizando os descritores: "coronavírus na gestação" e "COVID-19 na gestação". A busca ocorreu em abril de 2020. Resultados: Foram selecionados 26 artigos à qual emergiram três categorias: Diagnósticos de COVID-19 em gestantes e precauções; Cuidados com gestantes com COVID-19; Cuidados com fetos e neonatos. Os sinais e sintomas em mulheres grávidas com COVID-19 tendem a ser leves ou moderadas, possivelmente como resultado dos efeitos combinados do gênero, idade e estado imunológico da gravidez. De acordo com a literatura a principal via de parto escolhida para as gestantes acometidas pelo coronavírus é o parto normal. Em trabalhos de parto, a segunda fase deve ser, se possível, adiantada, pois a dificuldade respiratória da gestante pode ser agravar devido ao esforço e utilização de máscara. Considerações finais: Os cuidados com gestantes não diferem dos da população em geral, sendo necessário um enfoque nos sinais vitais e sintomatologia de infecção. As condutas devem ser baseadas primeiramente na condição obstétrica, visto que ainda não há evidências sobre a transmissão vertical.

Palavras-chave: Infecções por coronavírus, Gravidez, Cuidados de enfermagem.

\section{ABSTRACT}

Objective: Identify, synthesize and analyze the literature related to the care with COVID-19 in pregnancy. Methods: Integrative review in Medline and Epistemonikos databases using the descriptors: "coronavirus in pregnancy" and "COVID-19 in pregnancy". The search took place in April 2020. Results: Twenty-six articles were selected from which three categories emerged: Diagnosis of COVID-19 in pregnant women and precautions; Care of pregnant women with COVID-19; Care of fetuses and newborns. Signs and symptoms in pregnant women with COVID-19 tend to be mild or moderate, possibly as a result of the combined effects of gender, age and immune status of pregnancy. According to the literature, the main delivery route chosen for pregnant women affected by the coronavirus is the normal delivery. In labor, the second stage should, if possible, be advanced, as the pregnant woman's breathing difficulty may be aggravated by the effort and use of a mask. Final considerations: The care of pregnant women does not differ from that of the general population, and a focus on the vital signs and symptoms of infection is needed. The ducts should be based primarily on the obstetric condition, since there is still no evidence of vertical transmission.

Keywords: Coronavirus infections, Pregnancy, Nursing care.

\section{RESUMEN}

Objetivo: Identificar, sintetizar y analizar la literatura referente al cuidado con COVID-19 durante el embarazo. Métodos: Revisión integradora en las bases de datos Medline y Epistemonikos utilizando los descriptores: "coronavirus durante el embarazo" y "COVID-19 durante el embarazo". La búsqueda se realizó en abril de 2020. Resultados: Se seleccionaron 26 artículos y emergieron tres categorías: diagnósticos de COVID-19 en gestantes y precauciones; Atención a mujeres embarazadas con COVID-19; Cuidado de fetos y neonatos. Los signos y síntomas en mujeres embarazadas con COVID-19 tienden a ser leves o moderados,

1 Faculdades Integradas de Taquara (FACCAT), Taquara - RS. * E-mail: moniquevargas85@gmail.com SUBMETIDO EM: 7/2020

PUBLICADO EM: 10/2020 
posiblemente como resultado de los efectos combinados de sexo, edad y estado inmunológico del embarazo. Según la literatura, el principal modo de parto elegido para las embarazadas afectadas por el coronavirus es el parto normal. En el trabajo de parto, la segunda fase debe, si es posible, adelantarse, ya que la dificultad respiratoria de la embarazada puede agravarse por el esfuerzo y el uso de una mascarilla. Consideraciones finales: La atención a las mujeres embarazadas no difiere de la de la población general, lo que requiere un enfoque en los signos vitales y los síntomas de la infección. Los procedimientos deben basarse principalmente en la condición obstétrica, ya que aún no hay evidencia de transmisión vertical.

Palabras clave: Infecciones por coronavirus, Embarazo, Cuidado de enfermera.

\section{INTRODUÇÃO}

No final de 2019 na província de Wuhan, na China, um novo vírus foi descoberto, o SARS-CoV-2, sendo o principal causador de vários tipos de pneumonias. Também chamado de coronavírus ou Coronavirus Disease 2019 (COVID-19), ele atinge as vias respiratórias da pessoa acometida em que ocorre uma agressividade da resposta inflamatória na lesão pulmonar. A base patológica da doença refere-se ao acometimento das células pulmonares afetadas - pneumócitos tipo Il e as células endoteliais capilares. Devido ao grau da lesão pulmonar, a evolução compromete as trocas gasosas, ocorrendo um aumento do exsudato de componentes plasmáticos para o espaço alveolar.

Os diversos estudos histológicos referem-se a uma lesão alveolar difusa, com a formação da membrana hialina, infiltração mononuclear e dos macrófagos do espaço alveolar e consequentemente espessamento difuso da parede desses alvéolos (BOECHAT JL, et al., 2020). A sua manifestação clínica ocorre com o vírus sendo transmitido de humanos para humanos através das gotículas respiratórias e superfícies contaminadas. Em média o seu período de contaminação ocorre entre 5 a 6 dias, podendo se estender por mais tempo em alguns casos. Seus principais sinais e sintomas incluem febre, tosse, dispneia, mialgia, fadiga, sintomas respiratórios superiores e sintomas gastrointestinais como diarreia.

O quadro clínico, que é muito semelhante a uma Síndrome Gripal, pode apresentar-se de forma assintomático, com sintomas leves e mais graves, levando em alguns casos ao óbito. As pessoas com maior risco para a doença são aquelas com comorbidades associadas, entre elas: hipertensão arterial, diabetes mellitus, cardiopatia, disfunções respiratórias, câncer, obesidade, doenças renais, imunossupressos, transplantados e gestantes de alto risco (NARANJO BD, et al., 2020).

A infecção pode ocorrer em qualquer faixa etária, porém tem um maior índice de letalidade em pessoas mais idosas e com patologias prévias associadas. Desde o início da pandemia há uma preocupação com a infecção do vírus em gestantes, com a transmissão vertical, possíveis malformações, mas também em relação ao parto e a amamentação (SILVA C e TAVARES M, 2020).

Em relação a COVID-19 na gestação, os estudos são limitados e a quantidade de informações ainda são escassas. Vale ressaltar que o organismo da gestante sofre inúmeras modificações, como alterações fisiológicas e imunológicas, alterações das células imunológicas do pulmão, podendo levar a um pior prognóstico nos casos de pacientes com pneumonia pelo vírus (SILVA C e TAVARES M, 2020). Diante do exposto, essa pesquisa teve como objetivo identificar, sintetizar e analisar as literaturas referentes ao cuidado com COVID-19 na gestação.

\section{MÉTODOS}

O presente estudo trata-se de uma revisão integrativa (RI) da literatura proposta por Cooper H (1984), contendo as seguintes etapas segundo Mendes KDS et al. (2008) e Galvão CM (2003): 1. Identificação do tema e seleção da hipótese ou questão de pesquisa para a elaboração da revisão integrativa; 2. Estabelecimento de critérios para inclusão e exclusão de estudos/ amostragem ou busca na literatura; 3 . Definição das informações a serem extraídas dos estudos selecionados/ categorização dos estudos; 4. Avaliação dos estudos incluídos na revisão integrativa; 5. Interpretação dos resultados; 6 . Apresentação da revisão/ síntese do conhecimento. 
A investigação teve início após a consulta das seguintes bases de dados eletrônicas: Medical Literature Analysis and Retrieval System Online (MEDLINE) e EPISTEMONIKOS, através da utilização dos seguintes descritores, segundo Descritores em Saúde da Bireme (DeCs): "coronavírus na gestação" e "COVID-19 na gestação". A busca pelos artigos ocorreu no mês de abril de 2020. Para nortear a nossa pesquisa foi utilizada a seguinte questão norteadora: Quais são os principais cuidados de gestantes com COVID-19?

Foram considerados critérios de inclusão: artigos disponíveis na íntegra no idioma (português, inglês e mandarim), no período de dezembro de 2019 a abril de 2020 e que tivessem relação com os objetivos do trabalho. Foram considerados critérios de exclusão: artigos de base de dados não indexadas, teses e dissertações.

Os artigos encontrados tiveram seus resumos lidos e foram selecionados de acordo com os critérios de inclusão e exclusão definidos previamente. Os dados foram sistematizados e apresentados em um quadro sinóptico e discutidos a fim de contemplar os objetivos deste trabalho. Foram discutidas as principais questões encontradas nos materiais selecionados e a partir disso, foram formuladas categorias para apresentar os resultados e fazer uma reflexão sobre os achados da pesquisa. Em relação aos aspectos éticos foram respeitados os direitos autorais e o conteúdo, não havendo modificação dos mesmos.

\section{RESULTADOS E DISCUSSÃO}

Durante a pesquisa foram encontradas 135 produções nas bases de dados consultadas. Após validação dos artigos, a partir da leitura dos resumos, foram excluídos 53 artigos que se repetiam nas bases de dados e os demais que não respondiam à questão de pesquisa. O Quadro 1 apresenta as informações sobre os cruzamentos dos descritores e o número de artigos encontrados em cada base de dados.

\begin{tabular}{|c|c|c|}
\hline \multirow{3}{*}{ 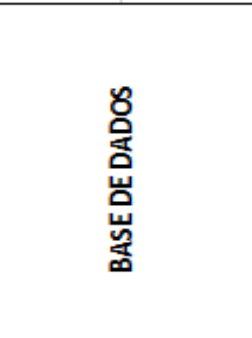 } & \multicolumn{2}{|c|}{ DESCRITORES } \\
\hline & \multicolumn{2}{|c|}{$\begin{array}{c}\text { Coronavírus na gestação e COVID-19 na } \\
\text { gestação }\end{array}$} \\
\hline & ENCONTRADOS & SELECIONADOS \\
\hline EPISTEMONIKUS & 7 & 1 \\
\hline \multirow[t]{3}{*}{ MEDLINE } & 75 & 25 \\
\hline & \multicolumn{2}{|c|}{ TOTAL ENCONTRADOS: 82} \\
\hline & \multicolumn{2}{|c|}{ TOTAL SELECIONADOS: 26} \\
\hline
\end{tabular}

Fonte: Cardoso MEV, et al., 2020.

Foram pré-selecionados 82 artigos e com o objetivo de responder à questão norteadora, respeitando os critérios de inclusão e exclusão da pesquisa, foram selecionadas apenas 26 publicações, após leitura na íntegra dos materiais encontrados (Quadro 2). A numeração dos artigos servirá de base para 0 referenciamento ao longo das categorias da análise.

Para análise do nível de evidência (Quadro 2), utilizou-se Galvão CM (2003), que cita que o conhecimento baseado em classificação de evidências proporciona ao enfermeiro melhoria na qualidade das avaliações dos resultados das pesquisas e consequentemente subsídios para tomada de decisão na prática clínica.

A classificação acontece de forma hierárquica, dependendo do delineamento de pesquisa, ou seja, da metodologia utilizada no estudo e ocorre da seguinte forma: Nível 1: revisões sistemáticas e metanálises de todos os ensaios clínicos randomizados e diretrizes clínicas; Nível 2: evidências oriundas de um ensaio clínico bem delimitado e randomizado; Nível 3: evidências obtidas de ensaios clínicos bem delimitados e sem randomização; Nível 4: evidências provenientes de estudo de coorte e caso controle bem delineado; Nível 5: evidências de revisões sistemáticas de estudos originais descritivos ou qualitativos; Nível 6: evidências de um único estudo descritivo ou qualitativo e Nível 7: evidências oriundas de opiniões de autoridades ou relatórios de comitês de especialistas. 
Quadro 2 - Publicações selecionadas, tipos de estudo e nível de evidência.

\begin{tabular}{|c|c|c|c|c|}
\hline Artigo & Autores & Título & Tipo de estudo & $\begin{array}{l}\text { Nível de } \\
\text { evidências }\end{array}$ \\
\hline A1 & KHAN AM, et al., 2020 & $\begin{array}{l}\text { COVID-19 infection during pregnancy: A systematic review to summarize } \\
\text { possible symptoms, treatments, and pregnancy outcomes }\end{array}$ & Revisão sistemática & 1 \\
\hline $\mathrm{A} 2$ & $\begin{array}{l}\text { SCHWARTZ DA e } \\
\text { GRAHAM AL, } 2020\end{array}$ & $\begin{array}{l}\text { Potential Maternal and Infant Outcomes from Coronavirus 2019-nCoV } \\
\text { (SARS-CoV-2) Infecting Pregnant Women: Lessons from SARS, MERS, } \\
\text { and Other Human Coronavirus Infections }\end{array}$ & Revisão sistemática & 1 \\
\hline A3 & SUN LL, et al., 2020 & $\begin{array}{l}\text { Perioperative Management of Cesarean Section for Pregnant Women With } \\
\text { Suspected or Confirmed COVID-19 }\end{array}$ & Revisão integrativa & 5 \\
\hline A4 & H QI, et al., 2020 & Safe Delivery for COVID-19 Infected Pregnancies & Revisão integrativa & 5 \\
\hline A5 & $\begin{array}{l}\text { LIANG H e ACHARYA G, } \\
\qquad 2020\end{array}$ & $\begin{array}{l}\text { Novel coronavirus disease (COVID-19) in pregnancy: What } \\
\text { clinical recommendations to follow? }\end{array}$ & Revisão integrativa & 5 \\
\hline A6 & CHEN D, et al., 2020 & $\begin{array}{l}\text { Expert consensus for managing pregnant women and neonates born to } \\
\text { mothers with suspected or confirmed novel coronavirus (COVID-19) } \\
\text { infection }\end{array}$ & Revisão integrativa & 5 \\
\hline A7 & POON LC, et al., 2020 & $\begin{array}{l}\text { Global interim guidance on coronavirus disease } 2019 \text { (COVID-19) during } \\
\text { pregnancy and puerperium from FIGO and allied partners: Information for } \\
\text { healthcare professionals }\end{array}$ & Revisão integrativa & 5 \\
\hline A8 & JIAO J, 2020 & $\begin{array}{l}\text { Under the epidemic situation of COVID-19, should special attention to } \\
\text { pregnant women be given? }\end{array}$ & $\begin{array}{l}\text { Recomendações de } \\
\text { integrativa de um estudo } \\
\text { descritivo }\end{array}$ & 6 \\
\hline A9 & CHEN S, et al., 2020 & $\begin{array}{l}\text { Clinical analysis of pregnant women with } 2019 \text { novel coronavirus } \\
\text { pneumonia }\end{array}$ & Estudo de coorte & 4 \\
\hline A10 & $\begin{array}{l}\text { RASMUSSEN SA, et al., } \\
2020\end{array}$ & $\begin{array}{l}\text { Coronavirus Disease } 2019 \text { (COVID-19) and pregnancy: what obstetricians } \\
\text { need to know }\end{array}$ & Revisão de especialistas & 7 \\
\hline A11 & $\begin{array}{l}\text { RASMUSSEN SA e } \\
\text { JAMIESON DJ, } 2020\end{array}$ & $\begin{array}{l}\text { Coronavirus Disease } 2019 \text { (COVID-19) and Pregnancy: what obstetricians } \\
\text { need to know Responding to a Rapidly Evolving Situation }\end{array}$ & Revisão de especialistas & 6 \\
\hline A12 & DASHRAATH, et al., 2020 & Coronavirus disease 2019 (COVID-19) pandemic and pregnancy & Revisão integrativa & 5 \\
\hline A13 & ZHI ZFCKZ, 2020 & Suggestions on Delivery Management of Pregnant Women With COVID-19 & Revisão integrativa & 7 \\
\hline A14 & WANG SS, et al., 2020 & $\begin{array}{l}\text { Experience of Clinical Management for Pregnant Women and Newborns } \\
\text { with Novel Coronavirus Pneumonia in Tongji Hospital, China }\end{array}$ & Coorte & 4 \\
\hline
\end{tabular}




\begin{tabular}{|c|c|c|c|c|}
\hline Artigo & Autores & Título & Tipo de estudo & $\begin{array}{l}\text { Nível de } \\
\text { evidências }\end{array}$ \\
\hline A15 & OMER S, et al., 2020 & Preventive measures and management of COVID-19 in pregnancy & Revisão integrativa & 5 \\
\hline A16 & LACOURSE S, et al., 2020 & $\begin{array}{l}\text { Importance of inclusion of pregnant and breastfeeding women in COVID-19 } \\
\text { therapeutic trials }\end{array}$ & Opiniões & 7 \\
\hline A17 & DONDERS F, et al., 2020 & ISIDOG Recommendations Concerning COVID-19 and Pregnancy & $\begin{array}{l}\text { Recomendações, } \\
\text { diretrizes }\end{array}$ & 7 \\
\hline A18 & FERRAZI EM, et al., 2020 & $\begin{array}{l}\text { COVID-19 Obstetrics Task Force, Lombardy, Italy: executive management } \\
\text { summary and short report of outcome }\end{array}$ & $\begin{array}{l}\text { Recomendações, } \\
\text { diretrizes }\end{array}$ & 7 \\
\hline A19 & SAHU KK, et al., 2020 & A twin challenge to handle: COVID-19 with pregnancy & Revisão integrativa & 5 \\
\hline A20 & ASHOKKA B, et al., 2020 & $\begin{array}{l}\text { Care of the Pregnant Woman with COVID-19 in Labor and Delivery: } \\
\text { Anesthesia, } \\
\text { Emergency cesarean delivery, Differential diagnosis in the acutely ill } \\
\text { parturient, Care } \\
\text { of the newborn, and Protection of the healthcare personnel }\end{array}$ & Revisão integrativa & 5 \\
\hline $\mathrm{A} 21$ & MIMOUNI F, et al., 2020 & $\begin{array}{l}\text { Perinatal aspects on the covid-19 pandemic: a practical resource for } \\
\text { perinatal-neonatal specialists }\end{array}$ & Revisão integrativa & 5 \\
\hline A22 & SUN LL, et al., 2020 & $\begin{array}{l}\text { Perioperative Management of Cesarean Section for Pregnant Women With } \\
\text { Suspected or Confirmed COVID-19 }\end{array}$ & Revisão integrativa & 5 \\
\hline A23 & WANG LS, et al., 2020 & $\begin{array}{l}\text { An Interpretation on Perinatal and Neonatal Management Plan for } \\
\text { Prevention and Control of SARS-CoV-2 Infection (2nd Edition) } 2020\end{array}$ & Revisão integrativa & 5 \\
\hline A24 & $\begin{array}{l}\text { Working Group for the } \\
\text { Prevention and Control of } \\
\text { Neonatal SARS-CoV-2 } \\
\text { Infection }\end{array}$ & $\begin{array}{l}\text { Perinatal and Neonatal Management Plan for Prevention and Control of } \\
\text { SARS-CoV-2 Infection (2nd Edition) }\end{array}$ & Revisão integrativa & 5 \\
\hline A25 & MULLINS E, et al., 2020 & Coronavirus in pregnancy and delivery: rapid review & Revisão integrativa & 5 \\
\hline A26 & PU J e LIU XX, 2020 & $\begin{array}{l}\text { Systematic perinatal management of the pregnant women and neonates } \\
\text { during the epidemic of COVID-19 }\end{array}$ & Revisão integrativa & 5 \\
\hline
\end{tabular}

Fonte: Cardoso MEV, et al., 2020. 
$\mathrm{Na}$ análise exploratória quanto ao ano de publicação, observa-se 25 publicações no ano de 2020, 1 publicação no ano de 2019. Quanto aos periódicos, as revistas que mais tiveram publicações foram à American Journal of Gynecology \& Obstetrics, Journal of Medical Virology e o International Journal of Gynaecology and obstetrics com 3 artigos.

A revista Chinese Journal of Contemporary Pediatrics teve dois 2 artigos. Demais revistas (Medrxiv, NCBI (National Center for Biotechnology information), BJOG, Acta Obstet Gynecol Scand, Drugs \& Therapy Perspectives, Clinical Infectious Diseases, Diagnostics, Journal of Perinatology, Chinese Medical Association, Chinese Society of Perinatal Medicine, Chinese Journal of Obstetrics and Gynecology, Current Medical Science, Am J Obstet Gynecol, Journal obstetrics, Journal obstet and gynecol), todos com uma publicação cada.

Após a leitura minuciosa dos artigos, foram elencadas 3 categorias para análise, são elas: 1- Diagnósticos de COVID-19 em gestantes e precauções, 2- Cuidados com gestantes com COVID-19 e 3- Cuidados com fetos e neonatos. As mesmas serão descritas a seguir. Abaixo, o Quadro 3 apresenta a relação dos artigos selecionados e usados em cada categoria.

Quadro 3 - Relação dos artigos selecionados por análise das categorias.

\begin{tabular}{|l|c|}
\hline \multicolumn{1}{|c|}{ Categorias } & Artigos \\
\hline $\begin{array}{l}\text { Diagnósticos de COVID-19 em } \\
\text { gestantes e precauções }\end{array}$ & A1, A9, A14, A15, A18, A19, A21. \\
\hline $\begin{array}{l}\text { Cuidados com gestantes com } \\
\text { COVID-19 }\end{array}$ & $\begin{array}{r}\text { A1, A2, A3, A4, A5, A6, A7, A8, A9, A10, A11, A12, A13, A14, } \\
\text { A15, A16, A17, A18, A19, A20, A24, A25, A26. }\end{array}$ \\
\hline Cuidados com fetos e neonatos & A2, A4, A5, A7, A9, A11, A12, A13, A17, A20, A24, A22. \\
\hline
\end{tabular}

Fonte: Cardoso MEV, et al., 2020

\section{Diagnósticos de COVID-19 em gestantes e precauções}

Dos artigos analisados, sete falam dos sinais e sintomas e diagnósticos relacionados às mulheres com COVID-19 na gestação. Alguns sinais e sintomas podem se sobrepor aos do novo coronavírus devido às modificações fisiológicas que ocorrem na gestação, fazendo com que muitas vezes dificulte ou atrase o diagnóstico (KHAN AM, et al., 2020; CHEN S, et al., 2020; WANG SS, et al., 2020; OMER S, et al., 2020; FERRAZI EM, et al., 2020; SAHU KK, et al., 2020; MIMOUNI F, et al., 2020).

Os sinais e sintomas em mulheres grávidas com COVID-19 tendem a ser leves ou moderadas, possivelmente como resultado dos efeitos combinados do gênero, idade e estado imunológico da gravidez, sugerindo que em uma área de alta prevalência de infecção, muitas mulheres grávidas podem ser positivas, mas assintomáticas (FERRAZI EM, et al., 2020). Costumam ser semelhantes à pacientes infectados em geral, sendo os principais sintomas febre, tosse e dificuldade respiratória, sendo a mialgia, dor de garganta e inapetência mais específicos nas gestantes (KHAN AM, et al., 2020).

Para o diagnóstico, além de observar os sintomas, deve-se monitorar os parâmetros laboratoriais incluindo hemograma completo, PCR (Polymerase Chain Reaction), gasometria arterial, painel metabólico, proteína C reativa, lactato, função renal, função hepática e enzimas cardíacas, juntamente com outros recursos de imagem como as tomografias computadorizadas do tórax. É importante salientar que a tomografia computadorizada do tórax é uma modalidade essencial para avaliar a pacientes com COVID-19 (CHEN D, et al., 2020; POON LC, et al., 2020; WANG SS, et al., 2020; SAHU KK, et al., 2020), com especial atenção e cuidado para os exames com contraste, visto que iodo pode atravessar a barreira placentária (A6).

Recomenda-se também, a realização da reação em cadeia da polimerase com transcrição reversa (RT PCR) da mucosa nasofaríngea e orofaríngea profunda nas condições clínicas apropriadas (SAHU KK, et al., 2020). Se o PCR não estiver disponível, um teste sorológico também pode ser usado para diagnóstico exame (OMER S, et al., 2020). 


\section{Cuidados com gestantes com COVID-19}

As gestantes sob suspeita ou diagnóstico de COVID-19 exigem muitos cuidados em relação ao diagnóstico e tratamento. Gestantes com apresentação clínica leve podem não requerer inicialmente internação hospitalar e o confinamento em casa pode ser considerado desde que isso seja possível logisticamente e que o monitoramento da condição da mulher possa ser assegurado sem comprometer a segurança de sua família (POON LC, et al., 2020).

É preciso proteger a paciente, o seu filho e as pessoas ao seu redor, desde familiares e profissionais da saúde, mesmo que não existam evidências de que as mulheres grávidas sejam mais suscetíveis a infecção por COVID-19 (POON LC, et al., 2020; RASMUSSEN SA, et al., 2020; LACOURSE S, et al., 2020; DONDERS F, et al., 2020). Tomar as precauções apropriadas são essenciais para evitar complicações, uma vez que se deve considerar que existem poucos estudos e protocolos específicos à essa temática (CHEN D, et al., 2020; POON LC, et al., 2020; LACOURSE S, et al., 2020; DONDERS F, et al., 2020). Dos 26 artigos analisados, à maioria (88\%) abordam os cuidados com as gestantes e COVID-19. A implementação de medidas preventivas é importante para evitar sua propagação (OMER S, et al., 2020).

Em relação ao uso de equipamentos de proteção individual (EPIs), primeiramente deve-se trabalhar com educação para os profissionais sobre a colocação e retirada de EPls e higienização das mãos. Os profissionais que prestam assistência a pacientes suspeitos ou confirmados para o novo coronavírus devem ser monitorados periodicamente. Os EPIs recomendados para uso dos profissionais seguem o padrão de proteção de três níveis: máscara N95, óculos ou protetor facial, gorro ou touca descartável, roupas de isolamento, capote ou avental impermeável, capas para sapatos, botas. O uso de dois pares de luvas de procedimento concomitantes é encorajado. Para as pacientes, a orientação é de que utilizem máscaras cirúrgicas ou máscaras N95 por todo o período de permanência nos serviços de saúde (SUN LL, et al., 2020; ZHI ZFCKZ, 2020).

Além da preocupação com o uso correto dos EPI's é importante salientar os cuidados com relação ao ambiente: implementar um fluxo de atendimento compatível com a redução de contaminação cruzada e fatores de risco de contaminação; manter internação em sala de parto, enfermaria ou quarto privativo; reduzir o número de pessoas circulantes em sala; realizar limpeza do ambiente conforme protocolo institucional minucioso e atualizado; e realizar a segregação do instrumental utilizado no trabalho de parto e parto, que devem ser identificados e processados separadamente (ZHI ZFCKZ, 2020; H QI, et al., 2020).

Para um manejo eficaz, as gestantes com suspeita de COVID-19 devem ser isoladas e depois transferidas para um hospital equipado com instalações de saúde suficientes e que, de preferência, sejam centros de maternidade específicos para pacientes com suspeita ou confirmação de COVID-19 (OMER S, et al., 2020; FERRAZI EM, et al., 2020). Independente do estado respiratório da mesma, ela deve ser posicionada em decúbito lateral esquerdo (CHEN D, et al., 2020). Caso não haja condições de transferir as pacientes para os centros de gestantes se faz necessário fornecer uma sala de parto específica para pacientes com COVID-19 (FERRAZI EM, et al., 2020).

Nos casos de gestantes internadas em enfermarias, a distância entre os leitos deve ser de pelo menos 1 metro, e que devam ser transferidas para a sala de operações e essa, deve ser equipada com pressão negativa e sinalizada como isolamento de COVID-19 para que os profissionais não façam paradas desnecessárias, aumentando assim, o risco de transmissão cruzada. A infecção afeta a função cardiopulmonar das gestantes, podendo acometer indiretamente a segurança intra-uterina fetal. Em alguns casos, pode-se exigir ressuscitação cardiopulmonar, reforçando a importância de um plano detalhado desse procedimento, garantindo assim a segurança da mãe e do filho (SUN LL, et al., 2020).

Para realização de cesárea em paciente diagnosticada e com suspeita, as duas podem ser realizadas na mesma sala desde que a suspeita seja feita antes da confirmada. Para gestantes estáveis é recomendada a utilização da anestesia peridural ou raquianestesia. As gestantes acometidas por pneumonia grave e SRAG, a anestesia intravenosa com intubação traqueal é indicada. A anestesia por inalação não é recomendada devido à maior dispersão do vírus no ar (SUN LL, et al., 2020; H QI, et al., 2020). 
Um dos estudos não recomenda a anestesia geral, pois aumenta o risco de transmissão do vírus devido a manipulação das vias aéreas no momento da intubação e extubação e também pelos efeitos que a anestesia causa no recém-nascido, como diminuição do tônus muscular e dificuldades na respiração (H Ql, et al., 2020).

Em relação às consultas de pós-parto, a maioria pode ser realizada remotamente, desde que a paciente não possua preocupações específicas que exijam exame pessoal. Certos aspectos, como a mama e ferida operatória podem ser avaliadas por vídeo ou fotos (POON LC, et al., 2020).

Quanto às alterações fisiopatológicas, é muito importante que a verificação dos sinais vitais da gestante seja rigorosa, com atenção especial para a frequência respiratória, saturação de oxigênio e temperatura corporal (H QI, et al., 2020; LIANG H e ACHARYA G, 2020; CHEN D, et al., 2020; POON LC, et al., 2020). Além da verificação rigorosa dos sinais vitais, a observação do aparecimento de sintomatologia sistêmica de infecção e alterações quanto a hemostasia se faz igualmente importantes (H QI, et al., 2020).

\section{Vias de parto e transmissão vertical}

A principal via de parto escolhida para as gestantes acometidas pelo coronavírus, indicada pela literatura, é o parto normal, devido a insegurança dos profissionais e falta de protocolos para esse procedimento. Em trabalhos de parto, a segunda fase deve ser, se possível, adiantada, pois a dificuldade respiratória da gestante pode ser agravar devido ao esforço e utilização de máscara. $O$ uso das banheiras é contra indicado, pois o contato com as fezes da parturiente pode aumentar o risco de transmissão para os profissionais envolvidos (POON LC, et al., 2020).

Por cesárea aumentar o tempo de internação das puérperas, o parto normal também é recomendado pela diminuição da exposição da mulher na instituição de saúde (KHAN AM, et al., 2020; H QI, et al., 2020). O acompanhante para a gestante deve ser desestimulado, visto o risco maior de transmissão (POON LC, et al., 2020).

Ao contrário de outros vírus estudados recentemente como o Ebola e Zikavírus, o novo coronavírus parece ter baixa ou não ter transmissão materno fetal no último trimestre da gravidez, não sendo encontrado no líquido amniótico, leite, placenta e nem nas vias aéreas do recém-nascido. No entanto, pode ocorrer transmissão por contato próximo do bebê com a mãe (SCHWARTZ DA e GRAHAM AL, 2020; LIANG H e ACHARYA G, 2020; CHEN D, et al., 2020; POON LC, et al., 2020).

Pacientes infectadas pelo COVID-19 não tem indicação absoluta de interrupção da gravidez, devendo sempre levar em conta a situação obstétrica, progressão da doença e estado do feto. Se a segurança de ambos estiver assegurada, a interrupção da gestação se dará pela idade gestacional (SCHWARTZ DA e GRAHAM AL, 2020; LIANG H e ACHARYA G, 2020; CHEN D, et al., 2020).

Embriões, fetos e placentas abortados de mulheres grávidas infectadas com COVID-19 devem ser tratados como tecidos infecciosos e devem ser descartados adequadamente e, se possível, deve-se realizar o teste desses tecidos para SARS-CoV-2 por RT-PCR (POON LC, et al., 2020).

\section{Uso de medicamentos e terapêutica}

Gestantes acometidas pelo COVID-19, por apresentarem na maior parte das vezes os mesmos sinais e sintomas da população em geral, acabam recebendo o mesmo tratamento, onde inclui oxigenoterapia em dispneias/baixa saturação, de suporte e de medicamentos para tratamento sintomático, antiinflamatórios e alguns antivirais, mesmo não sendo utilizado por todos (KHAN AM, et al., 2020; POON LC, et al., 2020; CHEN S, et al., 2020).

Os medicamentos específicos para o agravo ainda estão em fase de testes, porém alguns já estão sendo utilizados in vivo, com destaque para cloroquina e hidroxicloroquina. Esses podem causar danos fetais pois atravessam a barreira transplacentária e podem ser transferidos ao neonato através do leite materno durante a amamentação (KHAN AM, et al., 2020). O uso de antivirais na gestação é seguro, sendo os principais fármacos utilizados, o lopinavar e o ritanovir (LIANG H e ACHARYA G, 2020; CHEN D, et al., 2020; POON LC, et al., 2020). 
O uso de antibióticos para o tratamento da doença deve ser restringido apenas para as que apresentarem pneumonia por coronavírus. Medidas específicas e protocolos devem ser estabelecidos para esses casos, pois o tratamento em geral tem muitas semelhanças com outra pandemia anterior, como a Severe Acute Respiratory Syndrome (SARS) ou Síndrome Respiratória Aguda Grave (SRAG), onde além da oxigenoterapia, antibioticoterapia, medicamentos para tratamento de sintomas e antivirais, eles também utilizaram corticóides, o que não aconteceu ainda no tratamento da COVID-19. Há evidências que a utilização dos corticóides durante a gestação aumenta o risco de prematuridade, baixo peso ao nascer e pré eclâmpsia (KHAN AM, et al., 2020; LIANG H e ACHARYA G, 2020; CHEN D, et al., 2020; RASMUSSEN SA, et al., 2020).

\section{Cuidados com fetos e neonatos}

O monitoramento dos batimentos cardiofetais (BCFs) e/ou o ultrassom devem ser utilizados para avaliar a vitalidade fetal independentemente da idade gestacional. Recomenda-se um monitoramento mais avançado uma vez até que o feto atinja a viabilidade. A amniocentese deve ser considerada como parte de uma investigação diagnóstica (como avaliação de inflamação e infecção intra-amniótica), sendo que os riscos e benefícios de tais procedimentos devem ser discutidos com a paciente e com consentimento obtido (CHEN D, et al., 2020).

Para os partos de mães com a infecção, o obstetra e neonatologista devem trabalhar em conjunto, sendo que o último deve ser avisado com 30 minutos de antecedência do parto. Após o nascimento, deve-se preconizar o clampeamento precoce do cordão, secagem imediata e se o neonato apresentar boas condições clínicas, o mesmo deve ser transferido imediatamente para um quarto de isolamento e permanecendo em quarentena após a alta, durante 14 dias.

A amamentação direta não é recomendada para pacientes gravemente enfermas, sendo que a mesma deve ordenhar o leite e oferecer para o bebê através de um cuidador saudável (H QI, et al., 2020; CHEN D, et al., 2020; POON LC, et al., 2020; CHEN S, et al., 2020; RASMUSSEN SA, et al., 2020). Se a paciente apresentar sintomas leves ou for assintomática, o alojamento conjunto e amamentação podem ser realizados, mantendo todas as precauções necessárias. Como o vírus é transmitido através das gotículas, a utilização de máscara e rigorosa higiene de mãos para amamentar é necessário para a mãe (POON LC, et al., 2020; RASMUSSEN SA, et al., 2020).

\section{CONSIDERAÇÕES FINAIS}

Essa revisão integrativa evidencia que as gestantes podem ser consideradas um grupo de risco no que tange a susceptibilidade em adquirir o SARS-Cov-2 e desenvolver COVID-19, visto as mudanças fisiológicas próprias da gestação, bem como, não se faz claro ainda, aspectos sobre a probabilidade de transmissão vertical; o período de exposição da mãe e do feto e neonato ao vírus que se torna prejudicial para ambos; os efeitos dos medicamentos e outros tratamentos utilizados na gestação e período pós-parto, para o combate e controle da doença. Sabe-se que o primeiro trimestre é o período da gestação que demanda cuidados mais específicos e sensíveis durante o pré-natal. No entanto, os estudos analisados nessa revisão abordam gestantes de segundo e terceiro trimestres, deixando uma lacuna nessa discussão. Constatou-se que são necessários mais estudos como ensaios clínicos randomizados para a produção de evidências robustas com relação a essa temática.

\section{REFERÊNCIAS}

1. ASHOKKA B, et al. Care of the pregnant woman with coronavirus disease 2019 in labor and delivery: anesthesia, emergency cesarean delivery, differential diagnosis in the acutely ill parturient, care of the newborn, and protection of the healthcare personnel. American journal of obstetrics and gynecology, 2020;223(1), 66-74.

2. NARANJO BD, et al. Infección por SARS-CoV-2 en la paciente obstétrica: una perspectiva desde el cuidado crítico. Acta Colombiana de Cuidado Intensivo. 2020;20(2):98-107.

3. CHEN S, et al. Clinical analysis of pregnant women with 2019 novel coronavirus pneumonia. Journal of medical virology, 2020; 10.1002

4. CHEN D, et al. Expert consensus for managing pregnant women and neonates born to mothers with suspected or confirmed novel coronavirus (COVID-19) infection. International journal of gynaecology and obstetrics: the official organ of the International Federation of Gynaecology and Obstetrics, 2020; 149(2), 
5. COOPER H. The Integrative Research Review: A Systematic Approach Sage Publications: Beverly Hills, 1984,143 pp. Educational Researcher, 1986; 15(8), 17-18.

6. DASHRAATH, et al. Coronavirus Disease 2019 (COVID-19) Pandemic and Pregnancy. American Journal of Obstetrics and Gynecology. 2020; 222. 10.1016.

7. DONDERS F, et al. ISIDOG Recommendations Concerning COVID-19 and Pregnancy. Diagnostics (Basel, Switzerland), 2020; 10(4), 243.

8. FERRAZI EM, et al. COVID-19 Obstetrics Task Force, Lombardy, Italy: Executive management summary and short report of outcome. International Journal of Gynecology \& Obstetrics. 2020; 149. 10.1002

9. Galvão CM, et al. A busca das melhores evidências. Rev Esc Enferm USP. 2003 Dez; 37(4):43-50.

10. JIAO J. Under the epidemic situation of COVID-19, should special attention to pregnant women be given?. Journal of Medical Virology. 2020;10.1002

11. KHAN AM, et al. COVID-19 infection during pregnancy: a systematic review to summarize possible symptoms, treatments, and pregnancy outcomes, 2020.

12. LACOURSE S, et al. Importance of inclusion of pregnant and breastfeeding women in COVID-19 therapeutic trials. Clinical infectious diseases : an official publication of the Infectious Diseases Society of America, 2020.

13. LIANG H, ACHARYA G. Novel corona virus disease (COVID-19) in pregnancy: What clinical recommendations to follow?. Acta obstetricia et gynecologica Scandinavica, 99(4), 2020; 439-442.

14. MENDES KDS, et al. Revisão integrativa: método de pesquisa para a incorporação de evidências na saúde e na enfermagem. Texto \& Contexto - Enfermagem, 2008; 17(4), 758-764.

15. MIMOUNI F, et al. Perinatal aspects on the covid-19 pandemic: a practical resource for perinatal-neonatal specialists. J Perinatol 40, 820-826 (2020).

16. MULLINS E, et al. Coronavirus in pregnancy and delivery: rapid review. Ultrasound in obstetrics \& gynecology : the official journal of the International Society of Ultrasound in Obstetrics and Gynecology, 2020; 55(5), 586-592.

17. OMER S, et al.. Preventive measures and management of COVID-19 in pregnancy. Drugs \& Therapy Perspectives, 2020.

18. National Center for Health Care Quality Management in Obstetrics. Zhonghua fu chan ke za zhi, 2020; 55(3), 150-152.

19. POON LC, et al. Global interim guidance on coronavirus disease 2019 (COVID-19) during pregnancy and puerperium from FIGO and allied partners: Information for healthcare professionals. International journal of gynaecology and obstetrics: the official organ of the International Federation of Gynaecology and Obstetrics, 2020; 149(3), $273-286$.

20. Pu J, LIU XX. Systematic perinatal management of the pregnant women and neonates during the epidemic of COVID19. Zhonghua Fu Chan Ke Za Zhi. 2020;55(3):153-156.

21. H Ql, et al. Safe delivery for pregnancies affected by COVID-19. BJOG : an international journal of obstetrics and gynaecology, 2020; 127(8).

22. RASMUSSEN SA, et al. Coronavirus Disease 2019 (COVID-19) and pregnancy: what obstetricians need to know. American journal of obstetrics and gynecology, 2020; 222(5).

23. RASMUSSEN SA, JAMIESON DJ, 2020. Coronavirus Disease 2019 (COVID-19) and Pregnancy: Responding to a Rapidly Evolving Situation. Obstet Gynecol. 2020;135(5):999-1002.

24. SILVA C, TAVARES M. Da emergência de um novo vírus humano à disseminação global de uma nova doença Doença por Coronavírus 2019 (COVID-19), 2020.

25. SAHU KK, et al. A twin challenge to handle: COVID-19 with pregnancy. Journal of medical virology, $2020 ; 10.1002$.

26. SCHWARTZ DA, GRAHAM AL. Potential Maternal and Infant Outcomes from (Wuhan) Coronavirus 2019-nCoV Infecting Pregnant Women: Lessons from SARS, MERS, and Other Human Coronavirus Infections. Viruses, 2020; 12(2).

27. SUN LL, et al. Perioperative Management of Cesarean Section for Pregnant Women With Suspected or Confirmed COVID-19 2020;55(3):157-159.

28. WANG SS, et al. Experience of Clinical Management for Pregnant Women and Newborns with Novel Coronavirus Pneumonia in Tongji Hospital, China. Current medical science, 2020; 40(2), 285-289.

29. WANG LS, et al. An interpretation on perinatal and neonatal management plan for prevention and control of SARSCoV-2 infection, 2020;22(3):199-204.

30. Working Group for the Prevention and Control of Neonatal SARS-CoV-2 Infection in the Perinatal Period of the Editorial Committee of Chinese Journal of Contemporary Pediatrics (2020). Zhongguo dang dai er ke za zhi = Chinese journal of contemporary pediatrics, 2020; 22(3), 195-198. 